\title{
REPERCUSSÕES DA COVID-19 NA SAÚDE MENTAL DOS TRABALHADORES DE ENFERMAGEM
}

\section{REPERCUSSIONS OF COVID-19 IN THE MENTAL HEALTH OF NURSING WORKERS}

\section{REPERCUSIONES DEL COVID-19 EN LA SALUD MENTAL DE LOS TRABAJADORES DE ENFERMERÍA}

\author{
Emanuelli Mancio Ferreira da Luz ${ }^{1}$, Oclaris Lopes Munhoz ${ }^{2}$, Bruna Xavier Morais ${ }^{3}$, Patrícia Bitencourt Toscani Greco ${ }^{4}$, Silviamar \\ Camponogara ${ }^{5}$, Tânia Solange Bosi de Souza Magnago ${ }^{6}$
}

\section{RESUMO}

Objetivo: Refletir acerca das repercussões da Covid-19 na saúde mental dos trabalhadores de enfermagem. Método: Trata-se de um estudo de abordagem teórico-reflexiva. Fundamenta-se em aspectos conceituais sobre os agravos à saúde, da equipe de enfermagem em tempos de pandemia, na perspectiva da saúde do trabalhador. Resultados: O dimensionamento de recursos humanos insuficiente, a complexidade assistencial, o aumento da carga de trabalho, o medo de contaminação na utilização dos equipamentos de proteção individual e as condições insalubres dos serviços de saúde são situações que podem ocasionar adoecimento. Destas, o Estresse Ocupacional, a síndrome de Burnout, os Distúrbios Psíquicos Menores e o Sofrimento Moral podem estar acentuados, nesse período da pandemia, e repercutir, negativamente, na saúde física e psíquica da equipe de enfermagem. Conclusão: A promoção da saúde laboral tem sido alvo de políticas e estratégias governamentais e institucionais. Aos gestores cabe a proposição de medidas efetivas direcionadas a ambientes de trabalho saudáveis para que possam ser minimizadas as repercussões da pandemia na saúde dos trabalhadores de enfermagem.

Descritores: Pandemias; Infecções por Coronavirus; Saúde do Trabalhador; Enfermagem; Equipe de Enfermagem.

\begin{abstract}
Objective: to reflect on the repercussions of Covid-19 in the mental health of nursing workers. Method: this is a study with a theoretical-reflexive approach. It is based on conceptual aspects of health issues of nursing teams during pandemics, regarding workers' health. Results: the insufficient dimensioning of human resources, the complexity of caretaking procedures, the increased workload, the fear of contamination despite the use of personal protective equipment, and the unhealthy conditions in health services are situations that can cause illnesses. Of these, Occupational Stress, Burnout Syndrome, Minor Psychological Disorders and Moral Suffering may be accentuated, during this period of the pandemic, and have a negative impact on the physical and psychological health of the nursing team. Conclusion: occupational health promotion has been the target of governmental and institutional policies and strategies. Managers are responsible for proposing effective measures aimed at healthy work environments so that the repercussions of the pandemic on the health of nursing workers can be minimized.

Descriptors: Pandemics; Coronavirus Infections; Occupational Health; Nursing; Nursing, Team.
\end{abstract}

\section{RESUMEN}

Objetivo: reflexionar sobre las repercusiones del Covid-19 en la salud mental de los trabajadores de enfermería. Método: este es un estudio con un enfoque teórico-reflexivo. Se basa en aspectos conceptuales sobre los agravios en la salud del equipo de enfermería en tiempos de pandemia, desde la perspectiva de la salud del trabajador. Resultados: el dimensionamento insuficiente de los recursos humanos, la complejidad asistencial, el aumento de la carga de trabajo, el miedo a la contaminación durante el uso de los equipos de protección individual y las condiciones insalubres en los servicios de salud son situaciones que pueden causar enfermedades. De estos, el estrés ocupacional, el síndrome de Burnout, los trastornos psicológicos menores y el sufrimiento moral pueden acentuarse, en este período de pandemia, y tener un impacto negativo en la salud física y psicológica del equipo de enfermería. Conclusión: la promoción de la salud ocupacional ha sido el objetivo de las políticas y estrategias gubernamentales e institucionales. Los administradores son responsables de proponer medidas efectivas dirigidas a ambientes de trabajo saludables para que puedan ser minimizadas las repercusiones de la pandemia en la salud de los trabajadores de enfermería.

Descriptores: Pandemias; Infecciones por Coronavirus; Salud Laboral; Enfermería; Grupo de Enfermería.

${ }^{1}$ Mestre em Enfermagem. Doutoranda em Enfermagem pela Universidade Federal de Santa Maria. ${ }^{2}$ Mestre em Enfermagem. Doutorando em Enfermagem pela Universidade Federal de Santa Maria. ${ }^{3}$ Mestre em Enfermagem. Doutoranda em Enfermagem pela Universidade Federal de Santa Maria. ${ }^{4}$ Doutora em Enfermagem. Docente da Universidade Regional Integrada do Alto Uruguai e das Missões. ${ }^{5}$ Doutora em Enfermagem. Professora Associada do Departamento de Enfermagem da Universidade Federal de Santa Maria. ${ }^{6}$ Doutora em Enfermagem. Professora Permanente do Departamento de Enfermagem da Universidade Federal de Santa Maria.

\section{Como citar este artigo:}

Luz EMF, Munhoz OL, Morais BX, et al. Repercussões da Covid-19 na saúde mental dos trabalhadoresde enfermagem. Revista de Enfermagem do Centro Oeste Mineiro. 2020;10:e3824. [Access__]; Available in:__. DOI: http://doi.org/10.19175/recom.v10i0.3824 


\section{INTRODUÇÃO}

O atual contexto de saúde mundial está afetado pela pandemia causada pelo novo coronavírus, causador da Covid-19 (Corona Virus Desease-19). Há sete espécies de coronavírus humanos conhecidos, entre eles o Severe Acute Respiratory Syndrome Coronavirus-2 (Sars-Cov-2), causador da Síndrome Respiratória Aguda Grave. Este surgiu na cidade de Wuhan, na China, em 2019, e, a partir de então, ocasionou uma pandemia em larga escala. $O$ seu enfrentamento tem sido alvo prioritário da Organização Mundial da Saúde (OMS) ${ }^{(1)}$.

Nesse cenário, em 30 de janeiro de 2020, a OMS declarou que o surto da doença, causada pelo novo coronavírus, constitui uma Emergência de Saúde Pública de importância internacional, sendo o mais alto nível de alerta da Organização, conforme previsto no Regulamento Sanitário Internacional ${ }^{(2) .}$ A Covid-19 disseminou-se para diversos países, chegando ao Brasil, em 26 de fevereiro de 2020, quando o Ministério da Saúde informou o primeiro caso confirmado em território brasileiro. Em 11 de março de 2020, a Covid-19 foi caracterizada pela OMS como uma pandemia $^{(1)}$.

Com isso, faz-se necessário refletir acerca das relações trabalho-trabalhador no panorama dessa pandemia, especialmente dos trabalhadores de enfermagem, tendo em vista que atuam diretamente na prevenção e no cuidado integral dos pacientes infectados. $O$ ambiente de trabalho é permeado por fatores que interferem, negativamente, na saúde do trabalhador. Tal interferência pode comprometer a sua realização como profissional, bem como a sua capacidade para desenvolver as atividades laborais, o que pode repercutir na qualidade da assistência prestada ${ }^{(3)}$.

No cenário da Covid-19, observam-se mudanças significativas no que tange à realidade do trabalho. As organizações, sejam elas públicas ou privadas, tentam responder e se adaptar às exigências desse momento, tais como redimensionamento e reorganização de recursos humanos e materiais, elaboração e implementação de protocolos, entre outros. Já os trabalhadores de saúde estão expostos à acentuada pressão por produtividade e resultados, associados à intensificação das mudanças tecnológicas e ao risco de contaminação e adoecimento pela Covid-19. Também, pela constante ameaça de exposição de seus familiares ao contágio pelo vírus.
Ao reconhecermos que, mundialmente, ainda não há tratamento preventivo e de reabilitação efetivo para a Covid-19, como vacina ou tratamento medicamentoso, os holofotes voltam-se para aqueles que estão na linha de frente, dentre eles os trabalhadores de enfermagem. Para tanto, torna-se necessário um olhar atento e reflexivo a essa nova realidade laboral, a qual vem impondo um processo de trabalho modificado, por vezes carregado de anseios, estado de alerta e tensão. Este poderá resultar em danos à saúde física e psíquica dos trabalhadores de enfermagem ${ }^{(4)}$.

Diante desse contexto que permeia a pandemia, considera-se que, apesar do avanço do conhecimento acerca da saúde do profissional de enfermagem, persistem situações que podem predispor ao adoecimento laboral. Este pode decorrer de doenças ocupacionais, acidentes de trabalho, desgastes físicos e psíquicos ${ }^{(4)}$, especialmente $\mathrm{O}$ Estresse Ocupacional ${ }^{(5)}$, a Síndrome de Burnout ${ }^{(6)}$, os Distúrbios Psíquicos Menores (DPM) $)^{(7)}$ e o Sofrimento Moral $(\mathrm{SM})^{(8)}$. Nessa perspectiva, este estudo objetiva refletir acerca das repercussões da Covid-19, na saúde mental dos trabalhadores de enfermagem.

\section{MÉTODO}

Trata-se de um estudo de abordagem teórico-reflexiva. Fundamenta-se em aspectos conceituais acerca dos agravos à saúde mental da equipe de enfermagem, na perspectiva da saúde do trabalhador, da experiência dos autores na prática profissional e em pesquisas atuais relacionadas ao tema, como também nas notícias veiculadas sobre o impacto da pandemia.

Buscou-se discutir acerca das repercussões da pandemia Covid-19, especialmente no que concerne ao Estresse Ocupacional, ao Burnout, aos DPM e ao SM em trabalhadores de enfermagem. Os artigos analisados levaram à concretização de dois eixos temáticos: "Repercussões da Covid-19 na saúde mental dos trabalhadores de enfermagem" e "Estresse Ocupacional, Burnout, Distúrbios Psíquicos Menores e Sofrimento Moral: possíveis agravos à saúde mental dos trabalhadores como reflexo da pandemia Covid-19".

\section{RESULTADOS E DISCUSSÃO}

\section{Repercussões da Covid-19 na saúde mental dos trabalhadores de enfermagem}

O processo laboral dos trabalhadores de enfermagem, rotineiramente, é dinâmico e 
complexo. No contexto de uma pandemia, exigese (re)organização. Nessa perspectiva, compreender e discutir acerca dos processos de trabalho requer considerar os contextos históricos, culturais e estruturais ${ }^{(9)}$.

Historicamente, sabe-se que o sistema público de saúde brasileiro vivencia um subfinanciamento. Contudo, quando comparamos o cenário da Covid-19 às epidemias anteriores, percebemos que o momento ora vivenciado é mais grave. Neste, evidencia-se um aumento da necessidade de cuidados de alta complexidade para atender a milhares de pacientes infectados. Esse cuidado, realizado ao ser humano, é o objeto de trabalho primordial da Enfermagem. Essa categoria profissional representa o maior efetivo nas instituições de saúde $^{(9)}$ e, diante da pandemia, os trabalhadores de enfermagem passam a ser reconhecidos como protagonistas no enfrentamento da Covid-19.

No entanto, há um paradigma histórico relacionado à desvalorização, desprestígio, falta de proteção e invisibilidade dos trabalhadores de enfermagem em um contexto geral $^{(9-10)}$. Essa desvalorização fica evidente em estudo, ao apontar que um quarto, dos trabalhadores de enfermagem, não se sente acolhido, com respeito e gentileza, pelos demais colegas da área da saúde e $47,2 \%$ sentem-se desrespeitados pelos usuários e seus familiares ${ }^{(9)}$.

Além disso, os trabalhadores de enfermagem estão imersos em um processo estrutural e ambiente de trabalho permeado por condições que favorecem a exposição aos riscos de ordem biológica, física, química, ergonômica e psicossocial $^{(4)}$. Ademais, estão a mercê do processo de precarização, instabilidade trabalhista e financeira, flexibilização do trabalho com extensa carga horária e baixos salários, obrigando-os a duplas jornadas para complementação salarial $^{(9-10)}$.

Nesse momento, a preocupação no tocante à saúde dos trabalhadores de enfermagem está direcionada para o risco de exposição e contaminação pelo coronavírus, ou seja, para os protocolos com recomendações de medidas individuais de proteção, com a finalidade de conter a disseminação do vírus ${ }^{(11)}$. Estas incluem o controle ambiental, como a higiene e a desinfecção, a detecção precoce e a notificação dos casos, o distanciamento social e o uso dos Equipamentos de Proteção Individual (EPI) nos serviços de saúde ${ }^{(11-13)}$.
No contexto da pandemia, os trabalhadores de enfermagem da linha de frente estão em alto risco de adoecimento mental em razão da intensificação de algumas situações. Estas incluem o dimensionamento insuficiente e a complexidade assistencial, ocasionando o aumento da carga de trabalho, o receio de contaminação pelo inimigo invisível na utilização dos EPIs, a falta de estrutura e as condições insalubres dos serviços de saúde ${ }^{(12)}$. Somado a essas situações, há o isolamento familiar e social, o convívio diário com sofrimento e com as altas taxas de mortalidade dos colegas ${ }^{(12)}$.

Recomenda-se que os trabalhadores de saúde, que atuam, diretamente, no cuidado de pacientes portadores ou não de doença infectocontagiosa, façam o uso adequado do $\mathrm{EPI}^{(11,13)}$. Diante disso, há protocolos internacionais que regulamentam a paramentação e a desparamentação destes, com o intuito de evitar a contaminação ${ }^{(13)}$. Esses protocolos proporcionam maior segurança ocupacional aos profissionais de saúde, como também uma assistência de qualidade aos pacientes assistidos ${ }^{(11,13)}$.

No entanto, para segui-los, os trabalhadores são submetidos a extensas jornadas de trabalho, com vistas a reduzir o fluxo de pessoas no ambiente e a favorecer o uso racional do EPI, com consequente diminuição de gastos para as instituições ${ }^{(14)}$. As consequências físicas e psíquicas são nítidas para a saúde desses trabalhadores e incluem as lesões de pele, em região da face, pelo uso excessivo da máscara N95 e à retenção urinária, em razão de extensos períodos paramentados, fadiga/cansaço, entre outras $^{(14)}$.

Além disso, há o sofrimento dos trabalhadores de enfermagem relacionado ao cuidado de pacientes acometidos pela Covid$19^{(14)}$. Este é vivenciado no convívio com pacientes em isolamento, que não possuem a oportunidade de ter um familiar fisicamente presente, durante a internação e, ainda, em situações delicadas, como antes da sedação para procedimentos invasivos, como a entubação endotraqueal. Também, em razão do convívio com a impossibilidade de os familiares realizarem funerais, de acordo com sua tradição cultural, o que pode interromper o processo de luto ${ }^{(12)}$.

Desse modo, o trabalho realizado pela equipe de enfermagem, atuante na assistência aos pacientes acometidos pela Covid-10, pode resultar em adoecimento físico e psíquico ${ }^{(14)}$. Em 
resposta, observa-se que há políticas e estratégias oportunas e relevantes, de nível global, regional e local, direcionadas à promoção da saúde laboral, em tempos de pandemia ${ }^{(12,14)}$. Estas incluem as recomendações da OMS e do Ministério da Saúde, as políticas governamentais e institucionais, os serviços de telessaúde e os canais de comunicação direta para acesso aos serviços especializados de atenção à saúde do trabalhador $^{(12,14)}$. Ainda, destaca-se $\mathrm{o}$ monitoramento constante, dos trabalhadores de enfermagem infectados com a Covid-19, realizado pelo Observatório do COFEN ${ }^{(15)}$.

\section{Estresse Ocupacional, Burnout, Distúrbios Psíquicos Menores e Sofrimento Moral: possíveis agravos à saúde mental dos trabalhadores como reflexo da pandemia Covid- 19}

É nesse contexto de precarização, condições inadequadas e processos de trabalho reorganizados ${ }^{(9)}$ que se entende como necessário discutir os reflexos da pandemia no trabalho da enfermagem, bem como alguns dos possíveis agravos à saúde mental dos trabalhadores de enfermagem. Entre estes, o Estresse Ocupacional e a Síndrome de Burnout, os DPM e o SM.

\section{Estresse ocupacional e Síndrome de Burnout}

No que tange ao estresse, os trabalhadores de enfermagem se destacam entre as profissões que estão suscetíveis ao seu desenvolvimento ${ }^{(16)}$. Uma das formas desse agravo é a relacionada à interação do indivíduo com o ambiente laboral, denominada estresse ocupacional. Este está associado a altas demandas psicológicas, baixo controle sobre o trabalho e ao baixo apoio social recebido no ambiente de trabalho ${ }^{(5)}$. Ainda, relaciona-se às exigências mentais que implicam no trabalho do profissional. Sendo assim, quanto maior as exigências laborais, maior é a exigência psicológica do profissional ${ }^{(5,17)}$.

O estresse ocupacional é considerado um dos fatores que influenciam na atuação da equipe, durante a prestação dos serviços de saúde, visto que o excesso de demandas aumenta as exigências psicológicas ${ }^{(5,17)}$. Perante 0 cenário de pandemia, essas exigências podem ser potencializadas, ao passo que a equipe de enfermagem sofre com a tensão, a pressão do tempo, a interrupção das tarefas, entre outras ${ }^{(17)}$. Assim sendo, torna-se necessário desenvolver o controle sobre elas. A exemplo, tem-se o uso de habilidades (grau que o trabalho envolve a aprendizagem, repetitividade e criatividade) e a autoridade decisória (habilidade para a tomada de decisões no trabalho e influências na política gerencial) ${ }^{(5,17)}$.

Não obstante, a capacidade de controle sobre as demandas psicológicas pode ficar limitada diante das situações vivenciadas em uma pandemia. Além disso, quanto ao enfretamento dos estressores, vivencia-se a insegurança referente à possibilidade de falta de EPI, bem como de outros recursos materiais e humanos, a possível necessidade de isolamento de pessoas próximas e o medo de ser infectado pelo novo coronavírus ${ }^{(12-13)}$.

A acentuação do estresse ocupacional, no período atual da pandemia, entre os trabalhadores de enfermagem, pode associar-se à natureza do trabalho, o qual exige o cuidado direto aos pacientes acometidos pela Covid-19, com alto risco de contaminação. Destacam-se, ainda, como situações estressoras, o fato de não ter prazo determinado para o término da pandemia, a escassez dos EPI e a pressão, por parte de instituições, para a racionalização da utilização dos mesmos ${ }^{(13)}$.

Quando há exposição excessiva aos estressores, pode haver progressão para um processo crônico, levando o indivíduo a desenvolver a Síndrome de Burnout ${ }^{(6,18)}$. Essa síndrome pode ser desencadeada, principalmente, em indivíduos que desenvolvem seu trabalho atendendo a pessoas em contato direto $^{(19)}$, por exemplo, a equipe de enfermagem. Ainda, quando o indivíduo não consegue utilizar estratégias para enfrentar os estressores, acaba adoecendo $^{(3)}$. No contexto da pandemia, o uso de estratégias, seja de enfrentamento ou de fuga, pode estar fragilizado.

Partindo da concepção socioambiental da Síndrome de Burnout, existem fatores multidimensionais que consideram aspectos individuais, associados às condições e às relações do trabalho. Tais fatores se subdividem em três dimensões: o desgaste emocional, relacionado ao esgotamento físico, mental e emocional; a despersonalização, indicando que a personalidade do indivíduo está sofrendo alterações como consequência do seu trabalho; e a realização profissional, evidenciada pela (in)satisfação com as atividades laborais ${ }^{(6,18)}$.

Quando se olha para o cenário da Covid19, percebe-se que os trabalhadores de enfermagem estão fadados ao aumento de desgaste emocional e de despersonalização, bem 
como a uma baixa realização profissional, resultado do esgotamento físico e psíquico. Situações vivenciadas por plantões, muitas vezes dobrados, para atender às demandas da instituição em função da pandemia, pelo receio de contaminação, por falta de materiais e medo, por insegurança em relação ao trabalho e atuação, como também por vivenciarem a perda de vidas em massa, constituem-se em fatores que podem levar os trabalhadores de enfermagem à insatisfação profissional, expondo-os à Síndrome de Burnout.

Somado a isso, à medida que os trabalhadores de enfermagem se deparam com os estressores laborais, ficam sujeitos ao aumento da exaustão emocional ${ }^{(19)}$. Assim, muitas vezes, o indivíduo não percebe que está com a síndrome, ou seja, trabalha adoecido e, com isso, a interface entre saúde do trabalhador e segurança do paciente fica comprometida ${ }^{(3,6,19)}$.

\section{Distúrbios Psíquicos Menores}

Nesse contexto, destacam-se também os DPM, que são transtornos mentais leves e estão presentes entre os trabalhadores de enfermagem $^{(20)}$. Referem-se aos transtornos depressivos, de ansiedade ou somatização ${ }^{(7)}$, que não satisfazem a todos os critérios de adoecimento mental de acordo com a Classificação Internacional de Doenças (CID-10). Os DPM são caracterizados por um conjunto de sintomas não psicóticos, como insônia, fadiga, irritabilidade, esquecimento, diminuição da concentração e queixas somáticas $^{(7)}$.

Apesar da dificuldade em perceber esses sintomas como DPM, visto que podem ser atribuídos a diversas causas, tem-se o cenário no qual os trabalhadores de enfermagem desenvolvem as suas atividades ${ }^{(21)}$ e a exposição a situações que podem favorecer a ocorrência de $D P^{(20)}$. Nas instituições de saúde, as demandas psicológicas são significativas em decorrência do convívio com sofrimento e condições laborais precárias, jornadas extenuantes, falta de treinamento e, inclusive, insuficiência ou indisponibilidade de EPI. Esses fatores são predisponentes às alterações emocionais na equipe, provocando sintomas negativos, e potencialmente causadores do adoecimento psicológico ${ }^{(21)}$.

No contexto da pandemia Covid-19, há situações que podem levar ao desenvolvimento de sintomas de DPM. Estas incluem o aumento do número de casos confirmados, a insegurança quanto ao tratamento efetivo, a constante exposição ao vírus e o receio de constituir-se um potencial veículo de disseminação.

Além dessas situações, os trabalhadores de enfermagem convivem e presenciam o adoecimento e a morte de alguns colegas de profissão. Conforme o Conselho Federal de Enfermagem (Cofen) ${ }^{(15)}$, no Brasil, até 10 de julho de 2020, cerca de 249 trabalhadores, com diagnóstico de Covid-19, foram a óbito, com taxa de letalidade de 2,18\%. Ainda, 23.363 mil foram afastados das atividades laborais devido a Covid19.

Sendo assim, a iminência do adoecimento ocasiona ansiedade e insegurança entre os trabalhadores de enfermagem, que atuam no combate à Covid-19. E, com isso, demanda uma estrutura física e emocional exigente ${ }^{(20)}$ desses trabalhadores, resultado do contato contínuo com sofrimento, morte, jornadas múltiplas, sobrecarga de trabalho e baixa remuneração ${ }^{(21)}$. No enfrentamento à pandemia da Covid-19, essas exigências aumentam, podendo elevar a carga mental dos trabalhadores. Isso ocorre por conta da sobrecarga laboral, do crescente número de situações complexas que vivenciam, por meio da alta demanda de pacientes graves que, em sua maioria, necessitam de cuidados intensivos e exigem elevado desempenho da equipe de enfermagem ${ }^{(20)}$.

Essa carga mental elevada pode colaborar para que o trabalhador de enfermagem se mantenha nervoso ou preocupado, tenha cefaleia e redução na qualidade do sono. Tal situação pode causar cansaço e interferir na concentração. Esses sintomas, além de ocasionar intenso sofrimento psíquico, pode estar diretamente associado à redução da capacidade de trabalho e predispor à ocorrência de eventos adversos ${ }^{(20)}$.

\section{Sofrimento Moral}

Outro agravo à saúde dos trabalhadores de enfermagem é o SM, definido como um desequilíbrio psicológico que ocorre em virtude de uma situação em que o trabalhador necessita, por meio de seu julgamento moral, eleger uma conduta ética adequada a ser seguida. Entretanto, em decorrência de constrangimentos institucionais, o mesmo sente-se impedido de colocar em prática essa ação ${ }^{(8)}$.

Nesse contexto, os trabalhadores percebem que sua postura e seus valores estão sendo violados e, a partir disso, pode ocorrer o desencadeamento de aspectos negativos à saúde 
e bem-estar, podendo levá-los ao adoecimento $^{(22)}$. A frequência e a intensidade desses fatores contribuem para o cansaço e exaustão dos trabalhadores, interferindo, dessa forma, na qualidade da assistência prestada ${ }^{(23)}$.

A manifestação do SM pode ser expressa, por meio da desvalorização, invisibilidade e impotência. Além disso, o profissional pode se sentir incapaz de prosseguir no meio laboral e, muitas vezes, optar pelo abandono da profissão $^{(24)}$. Tais manifestações estão relacionadas ao contexto em que os trabalhadores estão inseridos, podendo associarse à redução da satisfação no trabalho e da capacidade de realizar as atividades ${ }^{(25)}$.

As formas de manifestações do SM associam-se à vivência de situações e particularidades do ambiente laboral, podendo ser emocionais ou físicas. Entre as formas emocionais, destacam-se as frustrações, o medo e os sentimentos de impotência ${ }^{(22,24)}$. Ao voltar-se ao cenário de pandemia da Covid-19, estas podem mostrar-se visíveis entre os trabalhadores de enfermagem, tendo em vista que os mesmos presenciam, diariamente, a superlotação de pacientes e a falta de leitos disponíveis para atendimento à saúde.

Em casos extremos, os trabalhadores de enfermagem e a equipe multiprofissional são forçados à realização de triagem, o que pode causar dilemas éticos com impacto traumático ${ }^{(13)}$. Essa triagem diz respeito à disponibilização de ventiladores mecânicos, que são equipamentos decisivos para a manutenção da via aérea do paciente acometido por Síndrome Respiratória Aguda Grave, para aqueles acometidos pela Covid-19.

Esse cenário propicia o sentimento de impotência, em que o trabalhador percebe a falta de poder na decisão dessas situações relacionadas à escassez de recursos e às dificuldades na gestão. Com isso, ele vivencia o SM e este pode ser agravado pelo risco de infecção, já que muitas instituições de saúde não possuem EPI suficientes ${ }^{(12)}$.

Outro aspecto, atrelado ao SM, é o estigma social, relatado pelos trabalhadores de enfermagem que atuam no cuidado aos pacientes com a Covid-19 ${ }^{(12)}$. Todavia, o que aqueles precisam é de suporte emocional e apoio social, pois têm que lidar com a ansiedade sobre sua condição, desconforto físico, isolamento e ser socialmente excluídos e separados das famílias ${ }^{(12)}$.
Ainda, entre as condições de trabalho, tem-se a escassez de EPI e de insumos. Tal situação é evidenciada na mídia mundialmente, em que a busca por esses insumos se torna cada vez mais acirrada entre os países ${ }^{(13)}$. Sendo assim, muitas vezes, os trabalhadores desenvolvem suas atividades em condições laborais inadequadas, o que se torna algo desafiador para eles, podendo resultar em medo, angústia e insegurança, bem como influenciar na qualidade do cuidado prestado $^{(12)}$.

Somado a isso, há a pressão por presenciar a piora do quadro clínico de pacientes, o medo de se contaminar, durante a paramentação e desparamentação dos EPI e a insegurança referente ao risco de contaminação dos familiares. A soma dessas situações pode desencadear manifestações físicas como crises de choro, insônia ou redução na qualidade do sono, perda de apetite, distúrbios gastrointestinais, entre outras ${ }^{(23,25)}$. No contexto atual da pandemia, reflete-se que o SM está intrínseco e também acentuado entre os trabalhadores de enfermagem.

$A$ insegurança com relação às condutas de tratamento e prevenção, a atenção aos familiares, as dificuldades diante da falta de equipamentos, de recursos humanos capacitados, como também as dúvidas quanto às condições do sistema de saúde absorver as demandas de pacientes graves em função da Covid-19, podem favorecer o SM desses trabalhadores. Essas angústias e incertezas podem fragilizar $o$ julgamento moral do profissional de enfermagem e, em contrapartida, ser empecilho para a realização de cuidado qualificado.

\section{CONCLUSÃO}

O estudo permitiu refletir a respeito das repercussões e possíveis agravos à saúde mental aos quais os trabalhadores de enfermagem possam estar expostos no enfrentamento da pandemia Covid-19. Destes, o Estresse Ocupacional, a Síndrome de Burnout, os DPM e o SM podem estar acentuados, nesse período da pandemia Covid-19. Da mesma forma, influenciar negativamente na saúde física e psíquica da equipe de enfermagem.

Percebe-se que este é um momento em que a Enfermagem possui uma visibilidade mundial e como uma categoria profissional fundamental no cuidado dos pacientes infectados. Para tanto, a promoção da saúde laboral tem sido alvo de políticas e estratégias 
governamentais e institucionais. Aos gestores cabe a (re)organização do trabalho e a proposição de medidas efetivas direcionadas a ambientes de trabalho saudáveis, para que possam ser minimizadas as repercussões da pandemia.

É evidente a necessidade de investimentos em acolhimento em saúde mental, medidas de monitoramento da sobrecarga e do estresse ocupacional, acompanhamento psicológico, e em redes de apoio social, para os trabalhadores de enfermagem, que busquem favorecer os relacionamentos interpessoais, como espaços de discussões coletivas e grupos multiprofissionais. Portanto, esforços imediatos devem ser empregados, em todos os níveis e pelas diversas áreas de conhecimento, visto que não há expectativas claras a respeito de quando ocorrerá o controle da pandemia Covid-19.

\section{REFERÊNCIAS}

1- World Health Organization (WHO). Novel coronavirus (2019-nCoV): Strategic preparedness and response plan. Geneva: WHO; 2020 [citado em 4 maio 2020]. Available in: https://reliefweb.int/report/world/2019-novelcoronavirus-2019-ncov-strategic-preparednessand-response-plan-draft-3

2- Ministério da Saúde. Portaria no 454, de 20 de março de 2020: declara, em todo o território nacional, o estado de transmissão comunitária do coronavírus (Covid-19). Diário Oficial da União 2020 1(59F):1.

3- Andolhe R, Barbosa RL, Machado EO, Costa ALS, Padilha KG. Stress, coping and burnout among intensive care unit nursing staff: Associated factors. Rev Esc Enferm USP 2015;49(Nesp):58-64. DOI: 10.1590/S0080623420150000700009

4- Carvalho DB, Araújo TM, Bernardes KO. Common mental disorders in primary health care workers. Rev Bras Saúde Ocup. 2016;41(17):1-13. DOI: 10.1590/2317.6369000115915

5- Karasek RA, Brisson C, Kawakami N, Houtman I, Bongers P, Amick B. The Job Content Questionnaire (JCQ): An instrument for internationally comparative assessments of psychosocial job characteristics. J Occup Health Psychol. 1998;3(4):322-355. DOI: 10.1037/10768998.3.4.322

6- Maslach C. Comprendiendo el burnout. Cienc Trab. 2009 [citado em 11 maio 2020]; 11(32):37-43. Available in: https://www.vitoria-
gasteiz.org/wb021/http/contenidosEstaticos/adju ntos/es/16/40/ 51640.pdf

7- World Health Organization (WHO). Depression and other common mental disorders: Global health estimates. Geneva: World Health Organization; 2017 [citado em 11 maio 2020]. Available in: http://www.who.int/mental_health/managemen t/depression/prevalence_global_health_estimate s/en/

8- Jameton A. A reflection on moral distress in nursing together with a current application of the concept. J Bioeth Inq. 2013;10(3):297-308. DOI: 10.1007/s11673-013-9466-3

9- Machado $M H$, Santos MR, Oliveira $E$, Wermelinger $M$, Vieira $M$, Lemos $W$, et al. Condições de trabalho da enfermagem. Enferm Foco 2016;7(nesp):63-71. DOI: 10.21675/2357707X.2016.v7.nESP.695

10- Melo CMM, Carvalho CA, Silva LA, Leal JAL, Santos TA, Santos HS. Nurse workforce in state services with direct management: Revealing precarization. Esc Anna Nery 2016;20(3):1-8. DOI: 10.5935/1414-8145.20160067

11- Leachi HFL, Ribeiro RP. Máscaras utilizadas pelos profissionais da saúde: o que é recomendado? Adv Nurs Health 2020;2(1):2-7. DOI: 10.5433/anh.2020v2.id39757

12- Ghebreyesus TA. Addressing mental health needs: An integral part of COVID-19 response. World psychiatry. 2020;(2):129-30. DOI: 10.1002/wps. 20768

13- World Health Organization (WHO). Rational use of personal protective equipment (PPE) for coronavirus disease (COVID-19): Interim guidance. Geneva: WHO; 2020 [citado em 23 abr 2020]. Available in: https://apps.who.int/iris/handle/10665/331498 14- Zhou Y, Zhou Y, Song Y, Ren L, Ng CH, Xiang Y, et al. Tackling the mental health burden of frontline healthcare staff in the COVID-19 pandemic: China's experiences. Psychol Med. 2020;2(1):1-5. DOI: 10.1017/S0033291720001622 15- Conselho Federal de Enfermagem (COFEN). Observatório da enfermagem 2020 [citado em 10 jul 2020]. Available in: https://observatoriodaenfermagem.cofen.gov.br/ 16- Pinto APCM, Silva MF, Azevedo ACB, Rodrigues CCFM, Salvador PTCO, Santos VEP. Estresse no cotidiano dos trabalhadores de enfermagem: Reflexos da rotina laboral hospitalar. Rev Enferm UFSM 2017;(6)4:548-58. DOI: $10.5902 / 2179769221779$

17- Alves MGM, Braga VM, Faerstein E, Lopes CS, 
Junger W. The demand-control model for job strain: A commentary on different ways to operationalize the exposure variable. Cad Saúde Pública 2015;31(1):1-5. DOI: 10.1590/0102$311 \times 00080714$

18- Leiter MP, Maslach C. Interventions to prevent and alleviate burnout. In: Leiter MP, Bakker AB, Maslach C (Eds). Burnout at work: A psychological perspective. New York: Psychology Press; 2014.

19- Dalcin L, Carlotto MS. Burnout syndrome in teachers in Brazil: Considerations for a research agenda. Psicol Rev. 2017;23(2):745-71. DOI: 10.5752/P.1678-9563.2017v23n2p745-770

20- Pinhatti EDG, Ribeiro RP, Soares MH, Martins JT, Lacerda MR. Minor psychiatric disorders in nursing: Prevalence and associated factors. Rev Bras Enferm. 2018;71(5):2176-83. DOI: 10.1590/0034-7167-2018-0028

21- Ferreira LL, Pinhatti EDG, Queiroz CKG, Ribeiro RP. Distúrbios psíquicos menores em trabalhadores de enfermagem de um bloco cirúrgico. Rev Baiana Enferm. 2019;33:1-10. DOI: 10.18471/rbe.v33.28279

22- Wachholz A, Dalmolin GL, Silva AM, Andolhe $\mathrm{R}$, Barlem ELD, Cogo SB. Moral distress and work satisfaction: What is their relation in nursing work? Rev Esc Enferm USP 2019;53:1-9. DOI: 10.1590/S1980-220X2018024303510

23- Wilson MA, Cutcliffe JR, Armitage $\mathrm{CNH}$, Eaton KN. Moral distress in the critical care air transport nurse. Nurs Outlook 2020;68(1):33-44. DOI: 10.1016/j.outlook.2019.07.003

24- Fruet IMA, Dalmolin GL, Bresolin JZ, Andolhe $\mathrm{R}$, Barlem ELD. Moral distress assessment in the nursing team of a hematology-oncology sector. Rev Bras Enferm. 2019;72(1):58-65. DOI: 10.1590/0034-7167-2017-0408

25- Burton M, Caswell H, Porter C, Mott S, DeGrazia M. Defined and described by neonatal and pediatric critical care nurses in a quaternary care free-standing pediatric hospital. Dimens Crit Care Nurs. 2020;39(2):101-109. DOI: 10.1097/DCC.0000000000000403
Nota: O presente estudo foi realizado com apoio da Coordenação de Aperfeiçoamento de Pessoal de Nível Superior - Brasil (CAPES) - Código de Financiamento 001.

Recebido em: 04/06/2020

Aprovado em: 15/07/2020

Endereço de correspondência:

Tânia Solange Bosi de Souza Magnago.

Universidade Federal de Santa Maria. Av. Roraima, 1000, Bairro Camobi. CEP: 97105-900 - Santa Maria/RS - Brasil.

E-mail:magnago.tania@gmail.com 